\title{
Tissue Classification of Noisy MR Brain Images Using Constrained GMM
}

\author{
Amit Ruf $^{1}$, Hayit Greenspan ${ }^{1}$, and Jacob Goldberger ${ }^{2}$ \\ 1 Department of Biomedical Engineering, Tel-Aviv University, Israel \\ ${ }^{2}$ School of Engineering, Bar-Ilan University, Israel
}

\begin{abstract}
We present an automated algorithm for tissue segmentation of noisy, low contrast magnetic resonance (MR) images of the brain. We use a mixture model composed of a large number of Gaussians, with each brain tissue represented by a large number of the Gaussian components in order to capture the complex tissue spatial layout. The intensity of a tissue is considered a global feature and is incorporated into the model through parameter tying of all the related Gaussians. The EM algorithm is utilized to learn the parameter-tied Gaussian mixture model. A new initialization method is applied to guarantee the convergence of the EM algorithm to the global maximum likelihood. Segmentation of the brain image is achieved by the affiliation of each voxel to a selected tissue class. The presented algorithm is used to segment 3D, T1-weighted, simulated and real MR images of the brain into three different tissues, under varying noise conditions. Quantitative results are presented and compared with state-of-the-art results reported in the literature.
\end{abstract}

\section{Introduction}

MRI images contain various noise artifacts, such as intra-tissue noise, inter-tissue intensity contrast reduction, partial-volume effects and others [1. Reviews on methods for brain image segmentation (e.g., 2] ) present the degradation in the quality of segmentation algorithms due to such noise, and recent publications can be found addressing various aspects of these concerns (e.g. 3]). Due to the artifacts present, classical voxel-wise based classification methods, such as Mixture of Gaussians modeling (e.g., [4]), may give unrealistic results, with tissue class regions appearing granular, fragmented, or violating anatomical constraints. Incorporating statistical spatial information via a statistical atlas [5], provides a means for improving the segmentation results. The co-registration of the input image and the atlas, a computationally intensive procedure, is critical in this scenario. Another conventional method to improve segmentation smoothness and immunity to noise is by using a Hidden Markov Random Field (HMRF), thus modeling neighboring voxels interactions [6].

This paper describes a robust, unsupervised and parametric method for the tissue segmentation of 3D MR brain images with a high degree of noise. The 
number of tissues is assumed given. Each tissue is modeled with multiple fourdimensional Gaussians, where each Gaussian represents a localized region (3 spatial features) and the intensity characteristic per region ( $\mathrm{T} 1$ intensity feature). The incorporation of the spatial information within the feature space is novel, as well as the use of a large number of Gaussians per brain tissue in order to capture the complicated spatial layout of the individual tissues. Note that models to-date use a single Gaussian per tissue type (e.g. [4]). An elaborate initialization scheme is suggested to link the set of Gaussians per tissue type, such that each Gaussian in the set has similar intensity characteristics with minimal overlapping spatial supports. The hypothesis is that all the Gaussians within the same class represent the same physical tissue with the same mean intensity and the same intensity covariance. It is assumed that the intensity inhomogeneity effect has been dealt with in a pre-processing phase. A detailed description of the proposed algorithm is provided in Sect 2. Experimental results are presented in Sect 3. The algorithm is validated on simulated brain as well as real brain volumes.

\section{The CGMM Segmentation Framework}

Given a brain volume, a 4-dim. feature vector is extracted for each voxel $v$. The main feature is the voxel intensity, denoted by $v^{I}$. In order to include spatial information, the $(x, y, z)$ position is appended to the feature vector. We use the notation $v^{x y z}=\left(v^{x}, v^{y}, v^{z}\right)$ for the three spatial features. Denote the set of the feature vectors by $\left\{v_{t} \mid t=1, \ldots, T\right\}$ where $T$ is the number of voxels in the image. In order to capture the complex spatial layout, we model the image using a mixture of many Gaussians: $f\left(v_{t} \mid \theta\right)=\sum_{i=1}^{n} \alpha_{i} f_{i}\left(v_{t} \mid \mu_{i}, \Sigma_{i}\right)$ such that $n$ is the number of components in the mixture model, $\mu_{i}$ and $\Sigma_{i}$ are the mean and the covariance of the $i$-th Gaussian component $f_{i}$, and $\alpha_{i}$ is the $i$-th mixture coefficient. The spatial shape of the tissues is highly non-convex. However, since we use a mixture of many components, each Gaussian component is modeling a small local region. Hence, the implicit convexity assumption induced by the Gaussian distribution is reasonable (and is empirically justified in the next section).

The intra variability of the intensity feature within a tissue (bias) is mainly due to artifacts of the MRI imaging process and once eliminated (via biascorrection schemes) is significantly less than the inter-variability among different tissues. It is therefore sufficient to model the intensity variability within a tissue by a single Gaussian (in the intensity feature). To incorporate this insight into the model, we further assume that each Gaussian is linked to a single tissue and all the Gaussians related to the same tissue share the same intensity parameters.

Technically, this linkage is defined via a grouping function. In addition to the GMM parameter set $\theta$, we define a parameter $\pi$ which is a grouping function $\pi:\{1, \ldots, n\} \rightarrow\{1, \ldots, k\}$ from the set of Gaussians to the set of tissues. We assume that the number of tissues is known and the grouping function is learned in the initialization step. The intensity feature should be roughly uniform in each Gaussian component spatial support, thus, its spatial and intensity features are assumed uncorrelated. These assumptions impose the following structure on the mean and variance of the Gaussian components: 


$$
\mu_{i}=\left(\begin{array}{c}
\mu_{i}^{\mathrm{xyz}} \\
\mu_{\pi(i)}^{\mathrm{I}}
\end{array}\right) \quad \Sigma_{i}=\left(\begin{array}{cc}
\Sigma_{i}^{\mathrm{xyz}} & 0 \\
0 & \Sigma_{\pi(i)}^{\mathrm{I}}
\end{array}\right) \quad i=1, \ldots, n
$$

where $\pi(i)$ is the tissue linked to the $i$-th Gaussian component and $\mu_{j}^{\mathrm{I}}$ and $\Sigma_{j}^{\mathrm{I}}$ are the mean and variance parameters of all the Gaussian components that are linked to the $j$-th tissue. We term the GMM model with the additional constraints Constrained-GMM (CGMM). The main advantage of the CGMM is the ability to combine in a tractable way, a local description of the spatial layout of a tissue with a global description of the tissue's intensity.

\subsection{Model Initialization}

We present a novel semi-supervised top-down initialization method that utilizes our knowledge about the number of tissues of interest. The initialization is a dual step procedure. In the first step K-means clustering is done based only on the intensity feature in order to extract a rough segmentation into 6 tissue classes (WM, GM, CSF, WM+GM, WM+CSF, GM+CSF) where combination of classes is due to partial volume effects. We assume the WM voxels to be of highest intensity, and the CSF voxels to be of lowest intensity. Voxels that belong to the four other classes are labeled as GM voxels. This rough but important initial clustering determines an initial group of voxels that belong to each tissue. We use this segmentation to calculate the approximate position of the Gaussians in each tissue. The next step is a top-down procedure. We iteratively split regions until we obtain convex regions that are suitable for Gaussian modeling. Each iteration of the spatial region splitting algorithm involves three steps: 1) A connected-components (CC) algorithm is used to define distinct regions which should be modeled by one or more Gaussians. Regions with less voxels than a user defined threshold are deleted and their voxels are marked as background noise, thus avoiding redundant Gaussians caused by noise; 2) Each region is encircled with the smallest ellipsoid possible1. If the volume inside the ellipsoid, that is not part of the region, is higher than a user defined threshold and the ellipsoid volume supports more voxels than the defined threshold value of minimum voxels per a region, the region is marked for further splitting; 3) A marked region is further split using K-means on the spatial features, into two distinct (not necessarily connected) subregions. The splitting algorithm iteratively proceeds as long as at least one region is marked for partitioning. Once the regions are determined, each region is modeled with a single Gaussian. The Gaussian's spatial parameters are estimated using the spatial features of the voxels supported by the region, while the intensity parameters is estimated using all the voxels supported by all the regions of the same tissue. Thus, Gaussians from the same tissue receive the same initial intensity parameter. Furthermore, each Gaussian is tagged with a label which indicates its tissue affiliation. Overall, the initialization process determines and fixes the grouping function $\pi$.

${ }^{1}$ Using the GBTß (Geometric Boundary Toolbox). 
Figure 1(a)-(e) illustrates the steps of the initialization process as applied to a $2 \mathrm{D}$ synthetic image with two tissues. A similar process is performed in $3 \mathrm{D}$ using a $3 \mathrm{D}$ ellipsoid instead of a $2 \mathrm{D}$ convex polygon for the convexity measure.

\subsection{Parameter Learning for the Constrained GMM}

Following initialization, an iterative maximum-likelihood (ML) learning process is conducted. Gaussians with the same tissue-label are constrained to have the same intensity parameters throughout. A modification of the standard (EM) algorithm for learning GMM is required, as shown in the following equations. The expectation step of the EM algorithm for the CGMM model is (same as the unconstrained version):

$$
w_{i t}=p\left(i \mid v_{t}\right)=\frac{\alpha_{i} f_{i}\left(v_{t} \mid \mu_{i}, \Sigma_{i}\right)}{\sum_{l=1}^{n} \alpha_{l} f_{l}\left(v_{t} \mid \mu_{l}, \Sigma_{l}\right)} \quad i=1, \ldots, n \quad t=1, \ldots, T
$$

We shall use the abbreviations:

$$
n_{i}=\sum_{t=1}^{T} w_{i t} \quad, \quad k_{j}=\sum_{i \in \pi^{-1}(j)} n_{i} \quad j=1, \ldots, k \quad i=1, \ldots, n
$$

such that $n_{i}$ is the expected number of voxels that are related to the $i$-th Gaussian component and $k_{j}$ is the expected number of voxels that are related to the $j$ th tissue. The maximization in the M-step is done given the constraint on the intensity parameters.

$$
\begin{aligned}
\alpha_{i} & =\frac{n_{i}}{n} & i & =1, \ldots, n \quad j=1, \ldots, k \\
\mu_{i}^{\mathrm{xyz}} & =\frac{1}{n_{i}} \sum_{t=1}^{T} w_{i t} v_{t}^{\mathrm{xyz}} & \Sigma_{i}^{\mathrm{xyz}} & =\frac{1}{n_{i}} \sum_{t=1}^{T} w_{i t}\left(v_{t}^{\mathrm{xyz}}-\mu_{i}^{\mathrm{xyz}}\right)\left(v_{t}^{\mathrm{xyz}}-\mu_{i}^{\mathrm{xyz}}\right)^{\top} \\
\mu_{j}^{\mathrm{I}} & =\frac{1}{k_{j}} \sum_{i \in \pi^{-1}} \sum_{t=1}^{T} w_{i t} v_{t}^{I} & \Sigma_{j}^{\mathrm{I}} & =\frac{1}{k_{j}} \sum_{i \in \pi^{-1}} \sum_{t=1}^{T} w_{i t}\left(v_{t}^{\mathrm{I}}-\mu_{j}^{\mathrm{I}}\right)^{2}
\end{aligned}
$$

The grouping function $\pi$ that links between the Gaussian components and the tissues is not altered by the EM iterations. Therefore, the affiliation of a Gaussian component to a tissue remains unchanged. However, since the learning is performed simultaneously on all the tissues, voxels can move between tissues during the iterations.

Figure 1(f) shows the CGMM model obtained for the synthetic image shown in Fig1(a) after seven EM iterations (following the top-down initialization). A $2 \sigma$ spatial projection of the region-of-support for each Gaussian in the model is shown (where different shades of gray represent the two distinct tissues present).

If the representation phase is a transition from voxels to clusters (Gaussians) in feature space, the segmentation process can be thought of as forming a linkage 


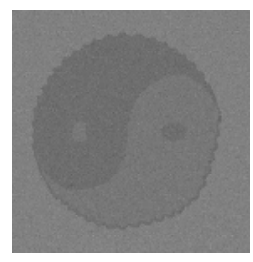

(a)

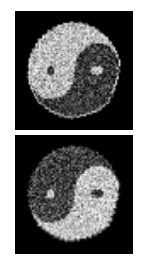

(b)

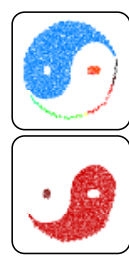

(c)

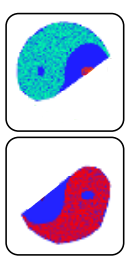

(d)

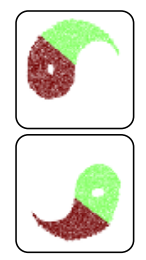

(e)

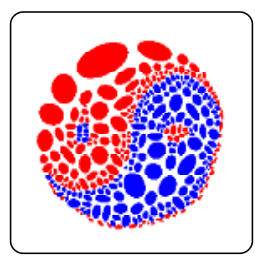

(f)

Fig. 1. Illustration of the Initialization process on a 2D synthetic image: (a) Input image; (b) Segmentation into two groups using the intensity feature only; (c) Connectedcomponents (CC) algorithm; (d) CC (region) is encircled with a convex polygon; (e) $\mathrm{CC}$ is clustered into two regions based on spatial features only; (f) Gaussian mixture modeling (seven EM iterations).

back from the feature space to the raw input domain. Each voxel is linked to the most probable Gaussian cluster, i.e. to the component of the model that maximizes the a-posteriori probability. The current model uses multiple Gaussians per tissue. Thus we need to sum over the posterior probabilities of all the identical tissue Gaussians to get the posterior probability of each voxel to originate from a specific tissue:

$$
\text { tissue-label }_{t}=\underset{j \in\{1, \ldots, k\}}{\arg \max } \sum_{i \in \pi^{-1}(j)} \alpha_{i} f_{i}\left(v_{t} \mid \mu_{i}, \Sigma_{i}\right) \quad t=1, \ldots, T
$$

such that tissue-label $t_{t} \in\{1, \ldots k\}$ is one of the tissues. The linkage of each voxel to a tissue label provides the final segmentation map.

\section{Experiments and Results}

In the following we present a set of experiments to validate the proposed framework. We used a severely noised normal T1 MR brain data set from the BrainWEB simulator repository 2 as well as 15 real normal T1 MR brain data sets from the Center for Morphometric Analysis at Massachusetts General Hospital repository 3 (hereon termed IBSR). An important pre-processing step is used to extract the brain region as well as to correct any intensity inhomogeneity (bias) present within the images. A variety of techniques are known in the literature. We use the algorithm proposed by Van-Leemput [ 6] as implemented in the EMS software package 4 . Next we applied our initialization step followed by EM iterations for CGMM. In our experiments a threshold of 250 voxels per region (Gaussian) is used in the initialization stage. In the first experiment we demon-

\footnotetext{
${ }^{2}$ http://www.bic.mni.mcgill.ca/brainweb/

${ }^{3}$ http://www.cma.mgh.harvard.edu/ibsr/

${ }^{4}$ http://www.medicalimagecomputing.com/EMS/
} 


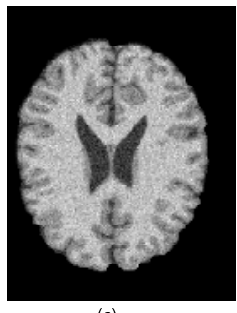

(a)

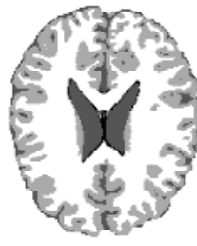

(b)

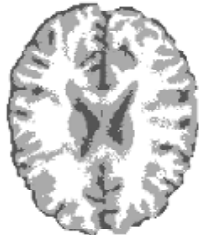

(c)

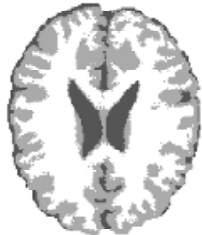

(d)

Fig. 2. (a) Slice number 95 from the BrainWEB normal brain simulator; (b) Groundtruth; (c) GMM-Without constraints; (d) CGMM-With constraints.

strate the performance of the CGMM framework as compared with regular EMbased modeling and segmentation. Figure 2 shows slice number 95 taken from the BrainWEB normal simulated brain volume, contaminated with $9 \%$ thermal noise artifact (relative to the white matter intensity). The Gaussian parameters were once learned using EM-based modeling (without intensity constraints) and once learned using the CGMM framework (with intensity constraints). Figure 2(c) shows the final segmentation using regular EM-based modeling. The segmentation result is noticeably distorted due to the high noise present. The segmentation results of the CGMM framework are shown in Fig 2 (d). A considerable improvement in the segmentation results can be seen. In the next set of experiments, a comparison is conducted with the well-known EM-based segmentation algorithm of Van-Leemput (hereon termed KVL) [6] implemented by the EMS software package. In the KVL implementation the statistic brain atlas of the SPM995 was normalized to the target brain volume. The EMS algorithm was setup to use the HMRF option as well as intensity inhomogeneity correction with 3D polynomial of order 4. Experiments were conducted on the simulated normal MR brain volume with very high thermal noise artifacts as well as on 15 real MR brain volumes from the IBSR. In the CGMM implementation, we used the EMS to remove any intensity inhomogeneity effects from the original real MR brain volumes as well as to extract only the brain tissues from the head volume.

We choose to compare the CGMM and the KVL algorithm with the volumetric overlap metric (the volumetric overlap metric is not claimed to be the best metric for medical image comparisons, but it is widely used). which was also used by [6. This metric is used to quantitatively measure the overlap between the automatic segmentation and the ground truth for each tissue and every algorithm. Denote by $V_{a e}^{k}$ the number of voxels that are assigned to tissue $k$ by both the ground truth and the automated algorithm. Similarly, let $V_{a}^{k}$ and $V_{e}^{k}$ denote the number of voxels assigned to tissue $k$ by the algorithm and the ground truth, respectively. The overlap between the algorithm and the ground truth for tissue $k$ is measured as: $2 V_{a e}^{k} /\left(V_{a}^{k}+V_{e}^{k}\right)$. This metric attains the value of one if both segmentations are in full agreement and zero if there is no overlap at all.

\footnotetext{
${ }^{5}$ http://www.fil.ion.ucl.ac.uk/spm/
} 
Table 1. Average and Std volumetric overlap results for CGMM on 16 brain volumes

\begin{tabular}{|c|c|c|c|c|c|}
\hline \multicolumn{3}{|c|}{ KVL } & \multicolumn{3}{c|}{ CGMM } \\
\hline WM & GM & CSF & WM & GM & CSF \\
\hline $86.0 \pm 2.0$ & $78.9 \pm 5.3$ & $20.7 \pm 16.6$ & $85.9 \pm 3.6$ & $79.5 \pm 5.7$ & $26.0 \pm 16.3$ \\
\hline
\end{tabular}
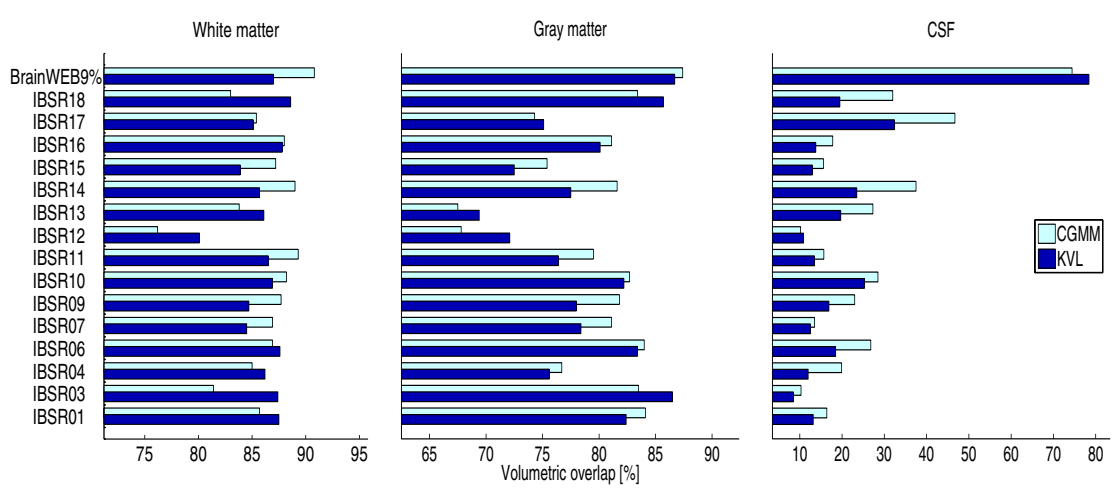

Fig. 3. Overlap metric of the CGMM algorithm (bright) and the KVL algorithm(dark) per each of the 16 volumes. Each graph shows a different tissue. The BrainWEB9\% is a simulated volume, where all the rest are real brain volumes.

Figure 3 shows the segmentation results of the CGMM algorithm (bright) and the KVL algorithm (dark). The CGMM performance improvement over the KVL is shown in most of the volumes and in all of the tissue types. However, CGMM's gain over the KVL is especially evident within the CSF tissue since the volumetric overlap tend to be more sensitive to outliers when dealing with small objects. Table 1 shows that the average results of the CGMM are slightly better than KVL in gray matter and even more significant in CSF. Furthermore, Fig 4 shows the smoothness in the segmentation output. The strength of the CGMM framework is more clearly evident in its robustness to noise and smooth segmentation results in increased noise levels.

\section{Discussion}

We present a fully automated, parametric, unsupervised algorithm for tissue classification of extremely noisy and low contrast MR images of the brain. The tissues are assumed to have been cleaned (preprocessed) of any intensity inhomogeneity. The algorithm was tested on different real brain volumes as well as heavily noised simulated brain volume. Quantitative comparison with KVL stateof-the-art algorithm in the presence of extremely noised images was performed and has shown that the CGMM presents better visual results in presence of high noise as well as better quantitative results - especially in CSF segmentation. This result holds with increased noise up to $9 \%$, a level that presents a challenge 


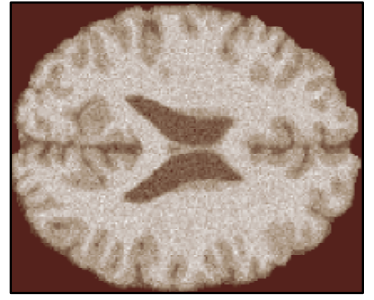

(a)

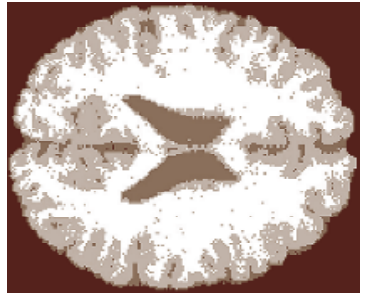

(b)

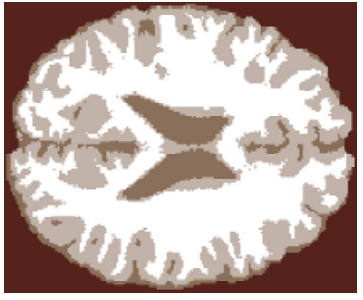

(c)

Fig. 4. Comparison of CGMM vs. KVL algorithm for segmentation of slice 95 from BrainWEB simulator with $9 \%$ noise level and no bias (a) Original image (b) KVL algorithm (c) CGMM algorithm

for most of the existing segmentation algorithms. The linkage of Gaussians has strong resemblance to using a HMRF model. The main difference is that the intensity information is linked adaptively and globally within the image, in contrast to a HMRF model that integrates information from the nearest neighbors only and in predetermined neighborhoods. These differences result in improved segmentation and decreased tissue region granularity in the presence of extreme noise. The CGMM is shown to provide a parametric framework good enough for segmentation without requiring a priori registration to a brain atlas, thus, it can be useful for abnormal brain segmentation, image registration as well as image retrieval. Currently we are working on an extension of the model to incorporate intensity inhomogeneities as well as to support multi-channel volumes.

\section{References}

1. Macovski, A.: Noise in MRI. Magn. Reson. Med. 36 (1996) 494-7

2. Pham, D.L., Xu, C., Prince, J.L.: Current methods in medical image segmentation. Annual Review of Biomedical Engineering 2 (2000) 315-337

3. Dugas-Phocion, G., Ballester, M.Á.G., Malandain, G., Lebrun, C., Ayache, N.: Improved em-based tissue segmentation and partial volume effect quantification in multi-sequence brain mri. In: MICCAI. (2004) 26-33

4. Kapur, T., Grimson, W.E., Wells, W.M., Kikinis, R.: Segmentation of brain tissue from magnetic resonance images. Med Image Anal. 1 (1996) 109-27

5. Prastawa, M., Gilmore, J., Lin, W., Gerig, G.: Automatic segmentation of neonatal brain mri. In: MICCAI. (2004) 10-17

6. Leemput, K.V., Maes, F., Vandermeulen, D., Suetens, P.: Automated model-based tissue classification of MR images of the brain. IEEE trans. on medical imaging $\mathbf{1 8}$ (1999) 897-908

7. Zhang, Y., Brady, M., Smith, S.: Segmentation of brain MR images through a hidden markov random field model and the expectation-maximization algorithm. IEEE trans. on medical imaging 20 (2001) 45-57 